\title{
Colonisation with toxigenic Corynebacterium diphtheriae in a Scottish burns patient, June 2015
}

\author{
A Deshpande ${ }^{1}$, T Inkster ${ }^{2}$, K Hamilton ${ }^{3}$, D Litt ${ }^{4}$, N Fry ${ }^{4}$, ITR Kennedy 5 , J Shookhye-Dickson ${ }^{5}$, RLR Hill ${ }^{4}$ \\ 1. Royal Alexandra Hospital, Glasgow, Scotland \\ 2. Queen Elizabeth University Hospital, Glasgow, Scotland \\ 3. Glasgow Royal Infirmary, Glasgow, Scotland \\ 4. Microbiology Reference Services, Public Health England, Colindale, United Kingdom \\ 5. Public Health Protection Unit, NHS Greater Glasgow and Clyde, Glasgow, Scotland
}

Correspondence: ashutoshdeshpande@nhs.net

Citation style for this article:

Deshpande A, Inkster T, Hamilton K, Litt D, Fry N, Kennedy I, Shookhye-Dickson J, Hill R. Colonisation with toxigenic Corynebacterium diphtheriae in a Scottish burns patient, June 2015. Euro Surveill. 2015;20(49):pii=30088. DOI: http://dx.doi.org/10.2807/1560-7917.ES.2015.20.49.30088

On 12 June 2015, Corynebacterium diphtheriae was identified in a skin swab from a burns patient in Scotland. The isolate was confirmed to be genotypically and phenotypically toxigenic. Multilocus sequence typing of three patient isolates yielded sequence type ST 125 . The patient was clinically well. We summarise findings of this case, and results of close contact identification and screening: 12 family and close contacts and 32 hospital staff have been found negative for $C$. diphtheriae.

\section{Case report}

On 9 June 2015 , a 20 year old female patient had routine swabs taken from the discharge of recent wounds, following reconstructive surgery. The samples subsequently grew Corynebacterium diphtheriae.

The patient had contracted severe $30 \%$ burns to her face, neck, chest, arms, and thighs as a child. She was followed up regularly by the plastic surgery team for management of burn contractures and skin grafts, and had no other relevant past medical history. On examination her wounds were not erythematous or ulcerated, and appearances were consistent with satisfactory healing.

The patient's childhood vaccination history was fully up to date as per the UK (UK) childhood vaccination schedule recommended [1], and included vaccination for $C$. diphtheriae infection.

Further questioning revealed no history of foreign travel at all in preceding years, or of close contacts who had travelled abroad, and there was no known animal contact. The patient had no clinical features of cardiological, neurological or cutaneous manifestations of $C$ diphtheriae. She reported a sore throat which was unremarkable on clinical examination with no membrane present. Following confirmation on 12 June of $C$. diphtheriae in swabs taken on 9 June, the patient was commenced on oral erythromycin 500mg, six hourly as per Public Health England (PHE) guidelines for the public health control and management of diphtheria. The treatment only started on 16 June, after some delays. The treatment continued for 14 days [2] and booster vaccinations were arranged. As the patient was very well, antitoxin was not administered.

Throat swabs and repeat skin swabs taken on 16 June, again grew toxigenic $C$. diphtheriae. Results were available on $19^{\text {th }}$ June and were identified by using MALDITOF and Hoyle's Tellurite agar.

Nose, wound and throat swabs were taken on 22 and 23 June. The results of the swabs were available on 26 June and were all negative for $C$. diphtheriae.

\section{Laboratory findings}

The organism was initially identified on the 12 June, by matrix-associated laser desorption ionisation time-offlight (MALDI-TOF) machine used for automated identification of microorganisms (Bruker); with a MALDI score of $99.9 \%$. This test was repeated and a Gram-stain demonstrated Gram-positive bacilli. Hoyle's Tellurite agar and the API Coryne system (bioMérieux) were subsequently used to confirm the identity of the strain as $C$. diphtheriae. The isolate was then sent to the PHE diphtheria national reference laboratory in Colindale, where it was confirmed as PCR-positive for the toxA gene, and phenotypically positive for toxin production by the Elek immunodiffusion test. The two later isolates from the throat and wound swabs were also confirmed as $C$. diphtheriae, tox- $A$-positive and Elek-positive. The genotypic relationship of all three $C$. diphtheriae isolates was characterised using the multilocus sequence typing (MLST) scheme described by Bolt et al. [3] comprising the seven $C$. diphtheriae housekeeping genes $\operatorname{atp} A, \operatorname{dnaE}, \operatorname{dnaK}$, fusA, leuA, odhA, and rpoB. Allelic 
Timeline for colonisation with toxigenic Corynebacterium diphtheriae in a Scottish burns patient, June 2015

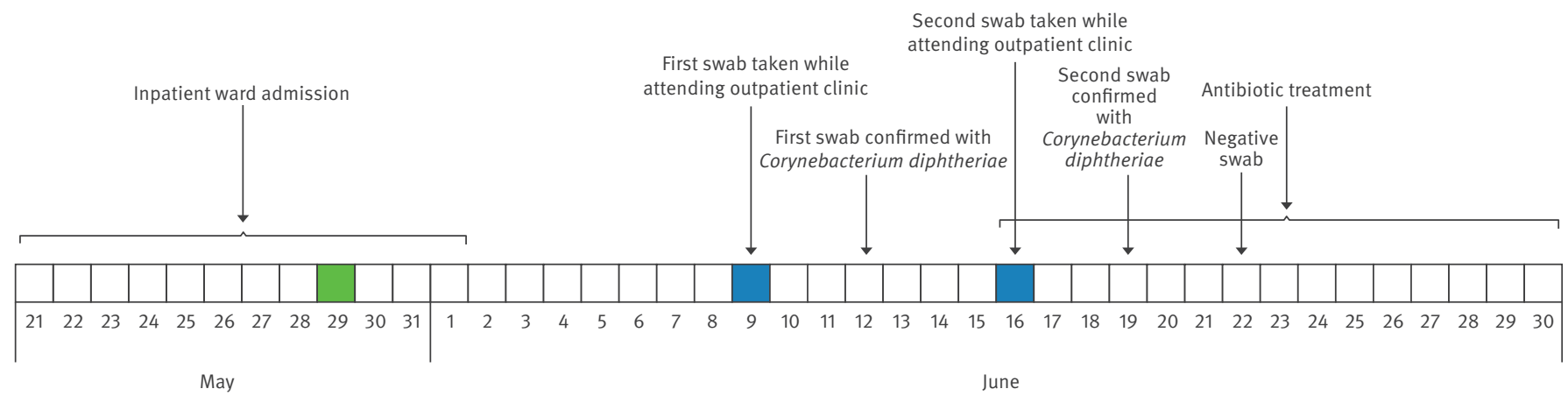

profiles and sequence type (ST) designations for each strain were obtained via the PubMLST database curated by the Pasteur Institut, Paris, France (http:// pubmlst.org/cdiphtheriae/). All three isolates had the alleleic profile $19,4,8,1,3,3,13$ corresponding to ST 125 .

Antibiotic susceptibility testing was undertaken using Clinical and Laboratory Standards Institute breakpoints [4] and the isolate tested sensitive to erythromycin, ciprofloxacin, meropenem, vancomycin, clindamycin, and clarithromycin. It was of intermediate sensitivity to penicillin. EUCAST breakpoints were not used due to lack of definitive breakpoints for $C$. diphtheriae.

\section{Contact tracing}

A timeline was prepared by our infection control team in conjunction with the clinical and public health teams, using the maximal incubation period of 10 days as a guide (Figure), with the caveat that the patient reported increased discharge from the wound before 31 May. The patient had spent most of this time as an outpatient at home, with the exception of being an inpatient for one day at the start of the lookback period, and presented to our clinic on 9 June 2015. Contact tracing was undertaken on all 12 family and other close contacts; this included friends, partner and relatives. Additionally, 32 hospital staff were identified as having had a relevant exposure during the patient's inpatient stay, such as being involved with changing wound dressings. Nose and throat swabs were taken from all of them, and Hoyle's Tellurite agar and MALDITOF techniques were used to screen specifically for the organism.

The vaccination histories of the patient's two year old child, family contacts as well as all hospital staff contacts, were checked and found to be complete as per UK recommendations. All identified family and close contacts and hospital staff were commenced on chemoprophylaxis with erythromycin at British National Formulary recommended doses, and excluded from work and encouraged to self-isolate until swab results were confirmed as negative. All contacts tested negative.

A memorandum was sent to all medical and nursing staff to enquire about recent foreign travel to exclude a hospital acquired infection.

The source of the organism remains unidentified.

\section{Discussion}

C. diphtheriae is an aerobic Gram-positive bacillus with a worldwide distribution. It causes diphtheria, a potentially life-threatening upper respiratory tract infection with a mortality rate of $5-10 \%$ in untreated cases $[2,4,5]$. As it is a vaccine preventable illness, it is now very rare in the European Union. Classic diphtheria is caused by bacteriophage-associated exotoxin-producing strains resulting in necrosis of infected cells and a classic fever, sore throat and tonsillar membrane. Non-toxigenic strains usually cause a milder illness or asymptomatic colonisation. Other manifestations of disease include cardiac syndromes (such as myocarditis), cutaneous lesions as well as neurological syndromes [5-7].

Humans are the main reservoir and transmit infection via respiratory droplets as well as direct contact. Respiratory and contact precautions are therefore required in an inpatient setting. The incubation period ranges from 1 to 10 days, and untreated individuals may be infectious for 2 to 4 weeks $[2,5]$. Treatment includes intravenous or oral macrolide therapy, intramuscular penicillin and antitoxin in severe illness.

Toxigenic $C$. diphtheriae is relatively rare in the UK and United States but prevalence is high in the Indian subcontinent and other parts of Asia, Eastern Europe, Africa, South and Central America. Numbers of toxigenic $C$. diphtheriae and $C$. ulcerans cases are one to six per year (2008-2014) in England [8]. Around 35 to 75 isolates of $C$. diphtheriae are submitted to the national reference laboratory per year (2010-2014) 
and the majority of these are subsequently shown to be non-toxigenic $[9,10]$.

Of 272 C. diphtheriae strains confirmed by the national reference laboratory from 2010 to $2014,69 \%$ were C. diphtheriae biovar gravis, $26 \%$ biovar mitis, and $4 \%$ biovar belfanti [11]. The strains from this case were all biovar mitis. The main risk factor for infection with toxigenic $C$. diphtheriae infection in the UK is travel to an endemic area or contact with others returning from such an area. For toxigenic $C$. ulcerans infection, the main risk factor is contact with animals including companion animals $[9,10]$. Neither the case nor the contacts had travelled abroad or had any animal contact.

This case demonstrates the importance of team work, and required close coordination and communication between clinical, infection prevention and control, public health, community health and reference laboratory teams. The organism was first identified by the microbiology team in a clinical laboratory who then contacted the general practitioner, clinical, infection control and public health teams. The ensuing multidisciplinary investigation required parallel contact tracing within and outwith the hospital settings, clinical assessments of the case and contacts, infection control investigations within the hospital, as well as close liaison with GPs of the case and contacts to guide chemoprophylaxis regimes as well as exclusion from work. Lines of communication between different multidisciplinary team members included phonecalls, emails as well as a teleconference and this was crucial in ensuring a coordinated and thorough investigation. Nevertheless, the case raised some questions; for example, whether responsibility for liaison with the general practitioner as well as the patient directly lay with the microbiology team or local health protection unit. Additionally, would a Standard Operating Procedure or protocol be warranted to guide teams when such situations arise?

Given the relative rarity of toxigenic diphtheria, it may be best to handle each case on an individual basis. With the advent of new molecular or rapid diagnostic technologies, such as the MALDI-TOF, it is possible that we may detect a greater number of any pathogen (not restricted only to $C$. diphtheriae) which hitherto may have gone unidentified, and this may have both clinical and epidemiological implications. Indeed, if a Gram stain had been done in the first place (which would traditionally have been the case), it might have just been discarded as a diphtheroid contaminant.

Due to the highly contagious nature of $C$. diphtheriae and its relative rarity in Scotland, where only two cases were reported between 2000 and 2013 [12], this case is of particular interest, especially as the strain involved was a toxin producing strain. The ST of the three isolates from this patient (ST 125) was first designated for an isolate of $C$. diphtheriae from 2009 from France. As of 24 November 2015, no other isolates with ST 125 have been diagnosed and the two closest profiles have four of seven alleles in common; ST $232(19,1,20,1,18,3,13)$ isolated in 2011 from Polynesia and ST $261(13,4,8,44,3,23,13)$ isolated in 2009 from a patient in England [8], from a patient with a chronic leg ulcer from a prosthetic knee infection. Interestingly, this latter patient had themselves lived on a Polynesian island. The source of the organism for the case described here remains unclear, and little is known of the true epidemiology in Scotland.

\section{Acknowledgements}

The authors would like to acknowledge Ms Asma Riaz, Biomedical Scientist, Glasgow Royal Infirmary; Mr Rob Anderson, Biomedical Scientist, Glasgow Royal Infirmary; Mr Stuart Kerr, Biomedical Scientist Glasgow Royal Infirmary; Mrs Laura Halley, Biomedical Scientist, Glasgow Royal Infirmary; Ms Fiona Graham, Staff Nurse, Glasgow Royal Infirmary; Mr Stuart Watson, Consultant Plastic Surgeon, Glasgow Royal Infirmary; Mrs Gillian Mills, Senior Infection Prevention \& Control Nurse, Glasgow Royal Infirmary; Mrs Jennifer Barrett, Infection Prevention \& Control Nurse, Glasgow Royal Infirmary.

\section{Conflict of interest}

None declared.

\section{Authors' contributions}

Dr Ashutosh Deshpande: first main author, wrote first draft and co-ordinated subsequent redrafts;

Dr Teresa Inkster: second main author, wrote first draft, helped with all redrafts, provided infection prevention control information; Ms Kate Hamilton: prepared timeline figure, provided information regarding hospital contact tracing and other aspects of infection prevention and control;

Dr David Litt: supported redraft of article and performed MLST analysis; Dr Norman Fry: supported redraft of article and molecular testing of isolates; $\mathrm{Dr}$ lain T R Kennedy: redrafted the article, provided epidemiological information including tracing of personal contacts and vaccination history, helped write discussion and management of case; Mrs Jacqueline Shookhye-Dickson: redrafted the article, provided epidemiological information including tracing of personal contacts and vaccination history, helped write discussion and management of case; Dr Robert L R Hill: helped to redraft the article and provide reference laboratory input.

\section{References}

1. Public Health England. Immunisation against infectious diseases: The Green Book. Available from: https://www.gov. uk/government/collections/immunisation-against-infectiousdisease-the-green-book [Cited 09/12/2015]

2. Diphtheria Guidelines Working Group. (March 2015). Public health control and management of diphtheria in England and Wales. Available from: https://www.gov.uk/government/ publications/diphtheria-public-health-control-andmanagement-in-england-and-wales [Cited 04/08/2015]

3. Bolt F, Cassiday P, Tondella ML, Dezoysa A, Efstratiou A, Sing $A$, et al. Multilocus sequence typing identifies evidence for recombination and two distinct lineages of Corynebacterium diphtheriae. J Clin Microbiol. 2010;48(11):4177-85. DOI: 10.1128/JCM.00274-10 PMID: 20844217

4. Clinical and Laboratory Standards Institute. (2015) Breakpoints available from: http://clsi.org/ [Cited 04/08/2015] 
5. Mandell B. Dolin. Principles and practice of infectious

diseases. 6th edition. Philadelphia: Elsevier Press 2001.

6. Moore LS, Leslie A, Meltzer M, Sandison A, Efstratiou A, Sriskandan S. Corynebacterium ulcerans cutaneous diphtheria. Lancet Infect Dis. 2015;15(9):1100-7. DOI: 10.1016/S14733099(15)00225-X PMID: 26189434

7. Galazka A. The changing epidemiology of diphtheria in the vaccine era.J Infect Dis. 2000;181(1) Suppl 1;S2-9. DOI: 10.1086/315533 PMID: 10657184

8. Both L, Collins S, de Zoysa A, White J, Mandal S, Efstratiou A. Molecular and epidemiological review of toxigenic diphtheria infections in England between 2007 and 2013.J Clin Microbiol. 2015;53(2):567-72. DOI: 10.1128/JCM.03398-14 PMID: 25502525

9. Public Health England. Diphtheria in England and Wales: 2014. Health Protection Report Volume 9 Number 18 Published on: 22 May 2015 https://www.gov.uk/government/uploads/system/ uploads/attachment_data/file/429678/hpr1815_dphthr.pdf [Cited 04/08/2015]

10. Public Health England. Infection Reports. Health Protection Report Volume 8 Number 12 Published on: 28 March 2014 https://www.gov.uk/government/uploads/system/uploads/ attachment_data/file/326923/hpr1214.pdf [Cited 04/08/2015]

11. Fry N. Respiratory and Vaccine Preventable Bacteria Reference Unit. Public Health England. Personal Communication. 04/08/2015.

12. Scotland HP. Annual Data Reports, 2012 \& 2013. http://www. hps.scot.nhs.uk/publichealthact/AnnualData.aspx [Cited $17 / 08 / 2015]$ 